(C) 2005 IEEE. Personal use of this material is permitted. Permission from IEEE must be obtained for all other uses, in any current or future media, including reprinting/republishing this material for advertising or promotional purposes, creating new collective works, for resale or redistribution to servers or lists, or reuse of any copyrighted component of this work in other works. 


\title{
Measurement and Modeling of Core Losses of Soft Magnetic Composites under 3D Magnetic Excitations in Rotating Motors
}

\author{
YouGuang GUO, Member, IEEE, Jian Guo ZHU, Senior Member, IEEE, Zhi Wei LIN, and Jin Jiang \\ ZHONG
}

\begin{abstract}
Soft magnetic composite (SMC) materials are especially suitable for construction of low cost high performance motors with three dimensional (3D) magnetic fields. The 3D finite element analysis conducted in the design of a claw pole transverse flux motor with an SMC core reveals very complicated $B$ (flux density) loci in the core when the motor rotates. In order to understand and account for the effects of the patterns of $B$ loci on motor parameters and performance such as core losses, a 3D magnetic property testing system was built for magnetic measurement on a cubic SMC sample. 3D rotational core loss models in SMC materials and rotating motors are developed.
\end{abstract}

Index Terms - 3D magnetic measurement, 3D rotational core loss, claw pole transverse flux motor, soft magnetic composite.

\section{INTRODUCTION}

Soft MAGNETiC composite materials and their Sapplication in electrical machines have achieved significant progress in the past decade due to the unique properties such as isotropic magnetic properties, very low eddy current loss, and nearly net-shape low cost fabrication process by using the powder metallurgical techniques [1]. Particularly, the magnetic isotropy creates crucial design advantages since radical topologies with three-dimensional (3D) magnetic fluxes can be exploited to obtain high motor performances, as the magnetic field constraints imposed by lamination technology can be avoided. SMC is suitable for construction of electrical machines with complex structures and 3D fluxes, e.g. claw pole and transverse flux motors.

Transverse flux motors (TFMs) have been a strong interest of research in the past years as they are capable of delivering very high specific torque provided that the number of poles is high and hence suitable for direct drive applications featuring high torque at low rotational speed. Because of the large armature leakage fields, the power factor of TFMs is quite low, especially the single-sided type [2]. The double-sided topology has a higher power factor and specific output, but all the designs reported are considered as too complicated to

This work was supported in part by the Australian Research Council under Large Grant No. A00104148.

The authors are with the Faculty of Engineering, University of Technology, Sydney, PO Box 123, Broadway, NSW 2007, Australia (e-mail: youguang.guo-1@uts.edu.au). manufacture [3]. To overcome this problem, a TFM with the claw pole geometry was developed in [4]. The claw pole TFM (CPTFM) features both the simplicity of the single-sided type and the high performance of the double-sided one. To improve the power factor, the claw poles are axially shortened for reducing the armature interpolar flux leakage. The decrease of the stator pole axial length will not necessarily reduce the stator winding flux produced by the rotor permanent magnets (PMs) when the flux-concentration structure is used.

Core loss calculation is a key factor in motor design and performance analysis. A significant amount of research work has been conducted on core loss measurement and modeling with one-dimensional (1D) alternating and two-dimensional (2D) rotating flux excitations by various researchers. The authors of this paper investigated the core losses in a claw pole PM motor with SMC stator core by the finite element analysis (FEA) of magnetic field, assisted by experimental data on SMC samples using a 2D magnetic property tester [5]. However, due to the rotation of magnetic domains the 1D and 2D measurements are all practical approximations under certain conditions [6]. 3D magnetic measurement and modeling are required for proper and complete understanding of magnetization process in the SMC materials.

In the CPTFM, the $\boldsymbol{B}$ loci in some parts are irregular loops in real 3D space. The core losses caused by such 3D B loci can be very different from that under $1 \mathrm{D}$ and $2 \mathrm{D}$ excitations, implying a direct need for 3D core loss investigation.

This paper presents our work about the measurement of $\boldsymbol{B}$ $\boldsymbol{H}$ curves and core losses on a cubic SMC sample by a 3D magnetic property tester [6], and the magnetic field analysis and core loss modeling in a CPTFM with SMC core.

\section{Claw Pole/Transverse Flux Motor}

Fig. 1 plots the layout and the magnetic field solution region of one pole pitch in a CPTFM with a PM rotor of a fluxconcentration structure. The simple toroidal winding is enclosed by an SMC core, which is molded in two halves. In the flux-concentration rotor, the PMs are magnetized tangentially and the fluxes produced by the two adjacent magnets aid in the SMC part between them and then flow to the stator via the airgap. The motor uses Somaloy ${ }^{\mathrm{TM}} 500$ $(+0.5 \%$ Kenolube) core [7] and NdFeB permanent magnets. 
The major dimensions and parameters are listed in Table 1.

TABLE I

\begin{tabular}{ll}
\multicolumn{2}{l}{ MAJOR DIMENSIONS AND PARAMETERS OF THE CLAW POLE TFM } \\
\hline \hline Quantity & Value \\
\hline Rated power (kW) & 2.2 \\
Rated speed (rpm) & 3000 \\
Rated frequency (Hz) & 300 \\
Number of phases & 3 \\
Stator core outer diameter (mm) & 110 \\
Middle airgap diameter (mm) & 56.5 \\
Airgap length (mm) & 1 \\
Magnet height (mm) & 14 \\
Stack axial length (mm) & 34 \\
Claw pole overlap ratio (\%) & 30 \\
\hline
\end{tabular}

Because the magnetic flux in a CPTFM is substantially 3D and the magnetic circuit is non-linear, the 3D FEA of magnetic field is necessary for accurate computation of motor parameters such as the back electromotive force, winding inductance, torque, and core losses.

The magnetic circuit of each stack (or phase) is basically independent. For each stack, because of the structural symmetry, it is only required to analyze the magnetic field in one pole pitch, as shown in Fig. 1. At the two radial boundary planes of one pole pitch, the magnetic scalar potential obey the half-periodical boundary conditions:

$\varphi_{m}(r, \Delta \theta / 2, z)=-\varphi_{m}(r,-\Delta \theta / 2,-z)$

where $\Delta \theta=30^{\circ}$ is the angle of one pole pitch.

Fig. 2 plots the computed flux density locus in the middle of (a) the stator yoke, (b) the claw pole, and (c) the flux concentration iron, respectively. It shows that the magnetic field in the CPTFM is really complex with various patterns when the rotor rotates.

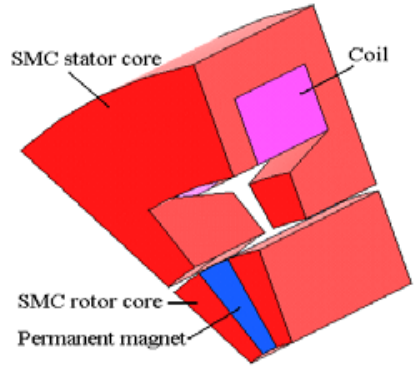

Fig. 1. Layout and FEA solution region of a CPTFM

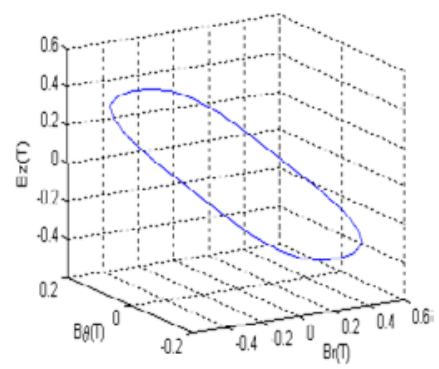

Fig. 2(b). $\boldsymbol{B}$ locus in the middle of the claw pole

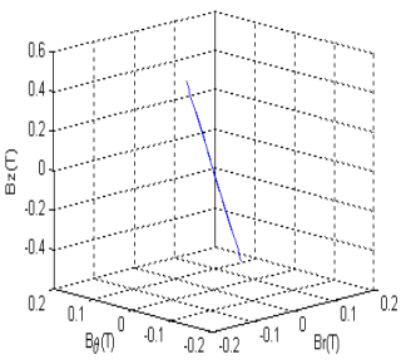

Fig. 2(a). $\boldsymbol{B}$ locus in the middle of the stator yoke

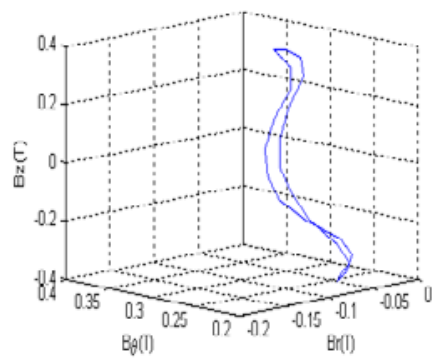

Fig. 2(c). $\boldsymbol{B}$ locus in the middle of the flux concentration iron

\section{3D Magnetic Property Measurement of SMC}

Due to the rotation of magnetic domains, the $\boldsymbol{B}$ locus in a

magnetic material is $3 \mathrm{D}$ in nature even under an alternating or 2D rotating magnetic excitation in a plane, no need to mention the 3D excitations in the CPTFM. Fig. 3 illustrates the block diagram of the 3D magnetic testing system and the tester magnetic circuit. Three groups of computer controlled excitation coils are employed to generate various $\boldsymbol{B}$ patterns including the ones in real 3D space, such as the patterns with the locus of the $\boldsymbol{B}$ vector tip forming an ellipsoidal or spherical surface. Fig. 4 shows the $\boldsymbol{B}-\boldsymbol{H}$ loops measured in an SMC cubic sample when a sinusoidal $\boldsymbol{B}$ with $50 \mathrm{~Hz}$ and various peak values is applied along the $\mathrm{X}$-axis, and the corresponding core losses. Figs. 5 and 6 show the $\boldsymbol{B}$ loci and the corresponding $\boldsymbol{H}$ loci, and the core losses when a circular $\boldsymbol{B}$ of $50 \mathrm{~Hz}$ is applied in the XOY plane or the YOZ plane.

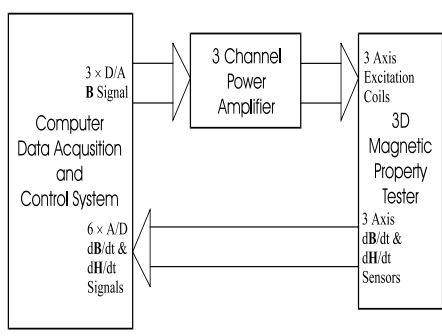

(a)

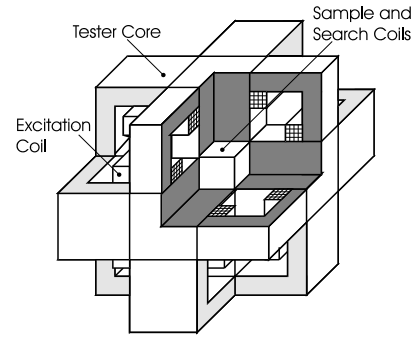

(b)
Fig. 3. (a) 3D magnetic property testing system; (b) cut away view of tester

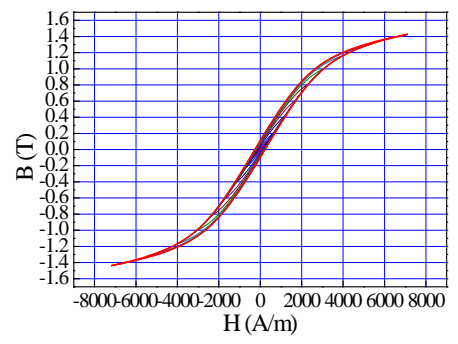

(a)

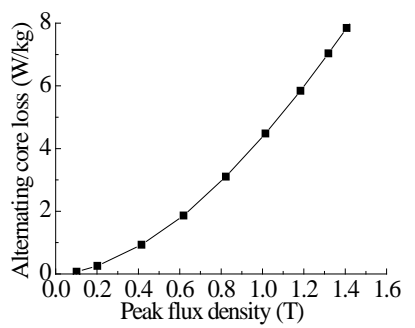

(b)
Fig. 4. (a) $\boldsymbol{B}-\boldsymbol{H}$ loops and (b) core losses with a sinusoidal $\boldsymbol{B}$ at $50 \mathrm{~Hz}$

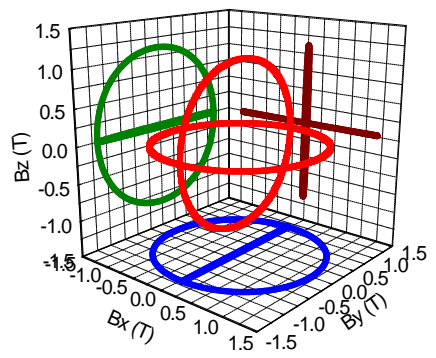

(a)

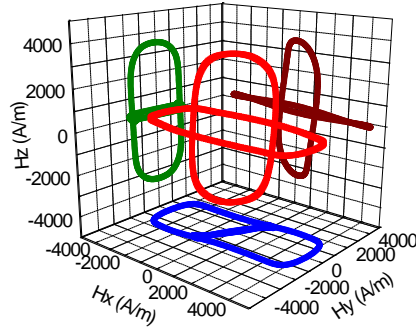

(b)
Fig. 5. (a) $\boldsymbol{B}$ loci and (b) corresponding $\boldsymbol{H}$ loci in $\mathrm{XOY}$ and YOZ planes

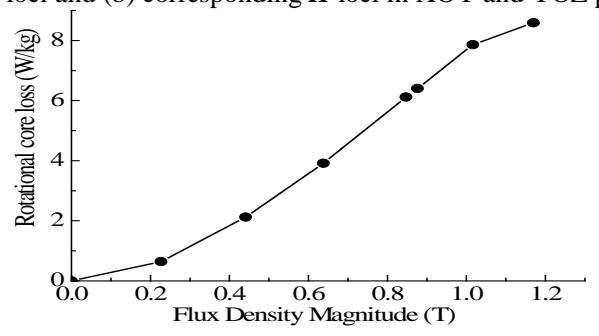

Fig. 6. Core losses with circular $\boldsymbol{B}$ at $50 \mathrm{~Hz}$

\section{MODELS OF 3D CORE LOSSES}

Under the condition that the magnetic field is uniform in the 
sample cross section, the specific core loss (in W/kg) in the sample due to a time-varying 3D magnetic flux density can be calculated according to the Poynting's theorem by

$P_{t}=\frac{1}{T \rho_{m}} \int_{0}^{T}\left(H_{x} \frac{d B_{x}}{d t}+H_{y} \frac{d B_{y}}{d t}+H_{z} \frac{d B_{z}}{d t}\right) d t$

where $T=1 / f$ is the time period, $f$ the frequency of magnetization, $\rho_{m}$ the sample mass density, and $H_{x}, H_{y}, H_{z}, B_{x}$, $B_{y}$, and $B_{z}$ are the $\mathrm{X}, \mathrm{Y}$ and $\mathrm{Z}$ components of $\mathbf{H}$ and $\mathbf{B}$.

When testing an SMC sample, the excitation flux density patterns are controlled either sinusoidally alternating along a specified orientation, or circularly rotating in a specified plane. In the sample, the alternating core loss can be modeled by

$P_{a}=C_{h a} f B_{P}{ }^{h}+C_{e a}\left(f B_{P}\right)^{2}+C_{a a}\left(f B_{P}\right)^{1.5}$

and the core loss with circular flux by

$P_{r}=P_{h r}+C_{e r}\left(f B_{P}\right)^{2}+C_{a r}\left(f B_{P}\right)^{1.5}$

where

$\frac{P_{h r}}{f}=a_{1}\left[\frac{1 / s}{\left(a_{2}+1 / s\right)^{2}+a_{3}^{2}}-\frac{1 /(2-s)}{\left[a_{2}+1 /(2-s)\right]^{2}+a_{3}^{2}}\right] \quad s=1-\frac{B_{P}}{B_{s}} \sqrt{1-\frac{1}{a_{2}^{2}+a_{3}^{2}}}$

The coefficients $C_{h a}, h, C_{e a}, C_{a a}, C_{e r}, C_{a r}, a_{1}, a_{2}, a_{3}$, and $B_{s}$ can be derived from the measured core losses on the cubic sample under various frequencies, $f$, and various peak flux densities, $B_{p}$ by curve fitting techniques.

The core loss with elliptically $\mathbf{B}$ can be predicted from the alternating and circularly rotating core losses by

$P_{t}=R_{B} P_{r}+\left(1-R_{B}\right)^{2} P_{a}$

where $R_{B}=B_{m i n} / B_{m a j}$ is the axis ratio of $\boldsymbol{B}, B_{m a j}$ and $B_{\min }$ are the major and minor axes of the elliptical $\boldsymbol{B}$ locus, $P_{r}$ and $P_{a}$ are the core losses with a circular $\boldsymbol{B}$ with $B_{m a j}=B_{\min }=B_{p}$ and an alternating $\boldsymbol{B}$ with peak value $B_{p}$.

In a rotating machine, the total core loss is the sum of the core losses in all elements. For any $\boldsymbol{B}$ locus in 3D space, its 3 components can be expended into Fourier series as

$$
B_{i}(t)=\sum_{k=0}^{\infty}\left[B_{i s k} \sin (2 \pi k f t)+B_{i c k} \cos (2 \pi k f t)\right]
$$

where $i=r, \theta, z, B_{r}, B_{\theta}$, and $B_{z}$ are the radial, circumferential and axial components of $\mathbf{B}$, respectively. Each of these harmonics consists of two parts: $\mathbf{n}_{\mathrm{sk}} \sqrt{{B_{r s k}}^{2}+B_{\theta \delta k}{ }^{2}+B_{z s k}{ }^{2}} \sin (2 \pi k f t), \mathbf{n}_{\mathrm{ck}} \sqrt{{B_{r c k}}^{2}+{B_{\theta c k}}^{2}+{B_{z c k}}^{2}} \cos (2 \pi k f t)$, where $\boldsymbol{n}_{\boldsymbol{s k}}$ and $\boldsymbol{n}_{\boldsymbol{c k}}$ are unit vectors, determined by $B_{r s k}, B_{\theta s k}$ and $B_{z s k}$, and $B_{r c k}, B_{\theta c k}$ and $B_{z c k}$, respectively. The two parts generally form an elliptical trajectory in a plane determined by $\boldsymbol{n}_{\text {sk }}$ and $\boldsymbol{n}_{\boldsymbol{c k}}$. The major axis $B_{k m a j}$ and the minor axis $B_{k \min }$ can be obtained by a coordinate rotation for the standard equation.

The total loss in an element can be obtained by summing up the contributions from these flux density harmonics. For each elliptically rotating flux density harmonic, the loss can be predicted from the corresponding alternating and rotational losses according to the axis ratio of the elliptical flux density. Therefore, the total loss is

$P_{t}=\sum_{k=0}^{\infty}\left[P_{r k} R_{B K}+\left(1-R_{B K}\right)^{2} P_{a k}\right]$

where $R_{B K}=B_{k m i n} / B_{k m a j}$ is the axis ratio of the k-th harmonic flux density, $P_{r k}$ is the purely rotational loss with flux density $B_{k m a j}$, and $P_{a k}$ is the alternating loss with $B_{p}=B_{k m a j}$.

Fig. 7 shows the calculated core losses in the CPTFM at different speeds. Unlike conventional machines in which the copper loss dominates, SMC motors have comparable core and copper losses. It is an interesting design point that the two losses take the same value for high motor efficiency.

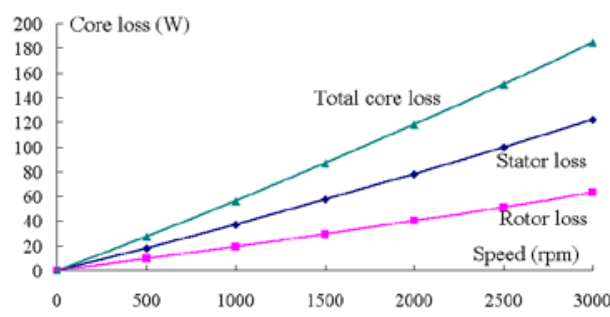

Fig. 7. Calculated core losses against rotor speed

\section{CONCLUSION}

The complexity of the $\boldsymbol{B}$ loci in a CPTFM with SMC core has been studied by 3D FEA of magnetic field, revealing the direct need for study about the 3D magnetic property of SMC. In this paper, core loss models with 3D rotating fluxes are proposed to calculate the core losses of rotating machines. The coefficients of the models are derived from the measurements on a cubic SMC sample by a 3D magnetic property tester.

\section{REFERENCES}

[1] "The latest development in soft magnetic composite technology," SMC Update, Reports of Höganäs AB, Sweden, 1997-2005. Available at http://www.hoganas.com/, see News then SMC Update.

[2] M.R. Harris, G.H. Pajooman, and S.M. Abu Sharkh, "The problem of power factor in VRPM (transverse flux) machines," in Proc. IEE Electric Machines and Drives Conf., Cambridge, UK, Sept. 1997, pp. 386-390.

[3] G. Henneberger and M. Bork, "Development of a new transverse flux motor," in Proc. IEE Colloq. New Topologies for PM Machine, June 1997, London, UK, pp. 1/1-6.

[4] C.P. Maddison, B.C. Mecrow, and A.G. Jack, "Claw pole geometries for high performance transverse flux machines," in Proc. Int. Conf. on Electrical Machines, Istanbul, Turkey, 1998, pp. 340-345.

[5] Y.G. Guo, J.G. Zhu, J.J. Zhong, and W. Wu, "Core losses in claw pole permanent magnet machines with soft magnetic composite core," IEEE Trans. Magn., Vol.39, Sept. 2003, pp. 3199-3201.

[6] J.G. Zhu, J.J. Zhong, Z.W. Lin, and J.D. Sievert, "Measurement of magnetic properties under 3-D magnetic excitations," IEEE Trans. Magn., Vol.39, Sept. 2003, pp. 3429-3431.

[7] "Soft magnetic composites from Hoganas Metal Powders SOMALOYT⿳ 500," Hoganas Product Guide, 1997. 